Urologe $2020 \cdot 59: 1512-1518$

https://doi.org/10.1007/s00120-020-01302-w

Online publiziert: 11. August 2020

(c) Der/die Autor(en) 2020

Philip Zeuschner · Stefan Siemer - Michael Stöckle • Matthias Saar

Klinik für Urologie und Kinderurologie, Universitätsklinikum des Saarlandes und Medizinische Fakultät der Universität des Saarlandes, Homburg/Saar, Deutschland

\title{
Die ersten 50 roboterassistierten Donornephrektomien
}

\author{
"Lessons learned"
}

\begin{abstract}
Die Nierenlebendspende stellt die beste Therapieoption bei terminaler Niereninsuffizienz dar. Sie stellt höchste Anforderungen an das Transplantationszentrum, nicht zuletzt deswegen, weil die Donornephrektomie an einem Gesunden erfolgt und Komplikationen schnell tragische Ausmaße haben können. Minimal-invasive Operationsverfahren nahmen 1995 mit der ersten laparoskopischen Donornephrektomie von Ratner et al. [25] Einzug in die Nierenlebendspende und führten zu steigender Akzeptanz.
\end{abstract}

Unerfreulicherweise konnten die zunehmenden Nierenlebendspenden den steigenden Bedarf an Spenderorganen jedoch nicht decken. Am 31.12.2019 standen 7148 Menschen allein in Deutschland auf der Warteliste für eine Nierenspende (vgl. Jahresbericht DSO für das Jahr 2019, s. Infobox), weltweit mehr als 120.000 Menschen.

Seit den 2000er-Jahren zählt die minimal-invasive Donornephrektomie (DN) zum operativen Standard und wird von den EAU Guidelines empfohlen [5]. Die operative Technik hat sich seit 1995 stetig fortentwickelt, auf die erste roboterassistierte $\mathrm{DN}$ (RDN) im Jahr 2000 folgte wenig später die erste laparoskopische Single-site-DN, bei der im LESS(„laparoendoscopic single site surgery")-Verfahren durch nur einen Schnitt operiert wird [12]. Neben handassistierten oder retroperitoneoskopischen Varianten existieren auch solche, bei denen versucht wird, nur durch natürliche Körperöffnungen, wie z.B. transvaginal, zu operieren [24].
Insgesamt konnte gezeigt werden, dass die minimal-invasive DN der offenen DN (ODN) überlegen ist [29]. Die laparoskopische DN (LDN) hat weniger Schmerzmittelbedarf, kürzere Hospitalisationsdauer, aber längere Operationsund warme Ischämiezeiten (WIZ). Trotzdem ist die LDN hinsichtlich des perioperativen Komplikationsrisikos und Graft-Überlebens der offenen DN nicht unterlegen. Beim direkten Vergleich von LDN und RDN ist die Evidenz geringer mit bisher nur zwei prospektiven, randomisiert kontrollierten Studien [3, 20]. Es wird häufig angeführt, die RDN habe noch weniger postoperative Schmerzen und weniger Blutverlust, aber längere Operations- und WIZ [28].

Die erste RDN in Deutschland erfolgte im Jahr 2007 in der Klinik für Urologie und Kinderurologie des Universitätsklinikums Homburg. Inzwischen wurden mehr als 50 Eingriffe durchgeführt, es handelt sich um die größte Kohorte einer deutschen urologischen Klinik. In einer retrospektiven Analyse wurde das perioperative Outcome analysiert und ein Follow-up der Nierenfunktion der Spender erhoben. Signifikante Prädiktoren für die Nierenfunktion bei Entlassung und im einjährigen Follow-up wurden mittels multivariabler Regressionsanalyse bestimmt.

\section{Material und Methoden}

Die ersten 50 RDN (2007-2019) wurden unizentrisch retrospektiv ausgewertet und ein Follow-up der Nierenfunktion erhoben. Relevante Patientencharakteristika wurden erfasst, wie das Verwandtschaftsverhältnis Spender/
Empfänger. Die Seite, szintigraphische Funktion (DTPA) und Gefäßversorgung der Spenderniere wurden erhoben. Operationsdauer, Blutverlust, warme (WIZ) und kalte Ischämiezeit (KIZ), Komplikationen nach Clavien-Dindo binnen 30 Tagen sowie die Verweildauer definierten das perioperative Outcome, das hinsichtlich der Entnahmeseite verglichen wurde. Die Nierenfunktion vom Spender wurde 5-10 Jahre postoperativ erhoben, sofern möglich, und nach $\mathrm{CKD}$ („chronic kidney disease“)-Stadien eingeteilt, als niereninsuffizient galt CKD-Stadium $\geq 3$ a. Signifikante Einflussfaktoren auf die Nierenfunktion und das Vorliegen einer Niereninsuffizienz wurden mittels Regressionsanalyse bestimmt.

\section{Operationsmethode}

Die RDN erfolgten entweder mit einem DaVinci $^{\circledR}{ }^{-}$Si- oder -X-System (Intuitive Surgical, Sunnyvale, Kalifornien, USA). Der Patient wurde ähnlich einer transperitonealen robotischen Nierenfreilegung in Seitenlage um etwa $10-15^{\circ}$ aufgeklappt. Die Trokare wurden pararektal

\begin{tabular}{|ll}
\hline \multicolumn{2}{|l}{ Abkürzungen } \\
\hline$C K D$ & "Chronic kidney disease \\
\hline$D N$ & Donornephrektomie \\
\hline$K I Z$ & Kalte Ischämiezeit \\
\hline$L D N$ & Laparoskopische Donornephrektomie \\
\hline$O D N$ & Offene Donornephrektomie \\
\hline$R D N$ & $\begin{array}{l}\text { Roboterassistierte Donornephrekto- } \\
\text { mie }\end{array}$ \\
\hline$W I Z$ & Warme Ischämiezeit \\
\hline
\end{tabular}


Tab. 1 Patienten- und Organcharakteris-

tika von 50 Nierenspendern, die einer RDN unterzogen wurden

\begin{tabular}{l|l}
\hline Variable & $\begin{array}{l}\text { Median (Range)/ } \\
\text { Häufigkeit }\end{array}$ \\
\hline $\begin{array}{l}\text { Alter (Jahre) } \\
\text { Geschlecht } 0^{7}\end{array}$ & $54(20-69)$ \\
\hline BMI (kg/m²) & $25,3(17,6-36,7)$ \\
\hline $\begin{array}{l}\text { Vorerkrankungen } \\
\text { Arterielle Hypertonie }\end{array}$ & $14(28 \%)$ \\
\hline $\begin{array}{l}\text { Diabetes } \\
\text { Nikotinabusus }\end{array}$ & $1(2 \%)$ \\
\hline $\begin{array}{l}\text { Abdominelle Vorope- } \\
\text { rationen }\end{array}$ & $26(10 \%)$ \\
\hline $\begin{array}{l}\text { Appendektomie } \\
\text { Cholezystektomie }\end{array}$ & $13(26 \%)$ \\
\hline Organspezifika & $4(8 \%)$ \\
\hline $\begin{array}{l}\text { Linke Seite } \\
\text { Funktion }\end{array}$ & $40(80 \%)$ \\
\hline $\begin{array}{l}\text { Gefäßversorgung } \\
\text { Eingefäßversorgung }\end{array}$ & $50 \%(39-57 \%)$ \\
\hline Mehrere Arterien & $6(12 \%)$ \\
\hline BMI Body Mass Index & \\
\hline
\end{tabular}

von kraniokaudal in einer geraden Linie eingelegt. Bei den ersten RDN erfolgte die Nierenentnahme durch einen Pfannenstielschnitt, später durch einen GelPort $^{\circledR}$-Trokar (Applied Medical, Rancho Santa Margarita, Kalifornien, USA) periumbilikal. Dazu werden während der Operation zwei Trokare durch den GelPort ${ }^{\circledR}$ geführt, sodass die Niere nach dem Absetzen der Gefäße und Abziehen der Porthülle problemlos mit der Hand geborgen werden kann. Zur Versorgung des Nierenstils wurden zunächst Staplersysteme verwendet, aufgrund schlechter Erfahrungen später Hem-O-Lok-Clips (Teleflex Medical, Morrisville, North Carolina, USA), die in der Regel doppelt zur Aorta und V. cava hin nebeneinander auf die Gefäße gesetzt werden. Ein Abrutschen von den Gefäßen wird durch eine periphere Gefäßnaht verhindert.

\section{Statistische Auswertung}

Kategoriale Variablen wurden in absoluter und relativer Häufigkeit angegeben, kontinuierliche als Median und Range. Fishers exakter, Mann-WhitneyU-, McNemar- und Wilcoxon-Rank-Test dienten zum Vergleich (un)verbundener

Tab. 2 Operatives Outcome von 50 RDN nach Entnahmeseite verglichen

\begin{tabular}{|c|c|c|c|c|}
\hline Variable & $\begin{array}{l}\text { Gesamt } \\
(n=50)\end{array}$ & $\begin{array}{l}\text { Links } \\
(n=40)\end{array}$ & $\begin{array}{l}\text { Rechts } \\
(n=10)\end{array}$ & $p$-Wert \\
\hline Operationszeit (min) & $223,5(127-363)$ & $223,5(127-363)$ & $219(177-318)$ & n.s. \\
\hline Blutverlust (ml) & $50(50-300)$ & $50(20-300)$ & $50(15-50)$ & n.s. \\
\hline WIZ (min) & $3(0,5-11)$ & $3(1-11)$ & $2(0,5-3)$ & $<0,05$ \\
\hline $\mathrm{KIZ}$ (min) & $76(22-202)$ & $76(22-177)$ & $117,5(31-202)$ & n.s. \\
\hline \multicolumn{5}{|l|}{ Komplikationen } \\
\hline Intraoperativ & $3(6 \%)$ & $2(5 \%)$ & $1(10 \%)$ & n.s. \\
\hline Postoperativ & $1(2 \%)$ & $1(2,5 \%)$ & - & n.s. \\
\hline \multicolumn{5}{|l|}{ Clavien-Dindo } \\
\hline $3 a$ & $1(2 \%)$ & $1(2 \%)$ & - & n.s. \\
\hline Verweildauer (Tage) & $5(2-12)$ & $5(3-12)$ & $6(2-11)$ & n.s. \\
\hline
\end{tabular}

\begin{tabular}{|c|c|c|c|c|c|c|c|c|}
\hline CKD-Grad & eGFR & Präoperativ & Postoperativ & $1 \mathrm{~J}$ & $2 \mathrm{~J}$ & $3 \mathrm{~J}$ & $5 \mathrm{~J}$ & $10 \mathrm{~J}$ \\
\hline 1 & $\geq 90$ & $48 \%$ & $2 \%$ & $4 \%$ & - & - & - & - \\
\hline 2 & $60-90$ & $53 \%$ & $32 \%$ & $48 \%$ & $44 \%$ & $33 \%$ & - & $100 \%$ \\
\hline $3 a$ & $45-59$ & - & $46 \%$ & $33 \%$ & $50 \%$ & $62 \%$ & $27 \%$ & - \\
\hline $3 b$ & $30-44$ & - & $2 \%$ & $15 \%$ & $6 \%$ & $5 \%$ & $73 \%$ & - \\
\hline 4 & $15-29$ & - & - & - & - & - & - & - \\
\hline 5 & $<15$ & - & - & - & - & - & - & - \\
\hline$n=$ & - & 50 & 50 & 27 & 18 & 21 & 11 & 2 \\
\hline
\end{tabular}

Stichproben. Bei der logistischen und linearen Regressionsanalyse wurden Kovariablen nur in die multiple Regression eingeschlossen, wenn sie auch univariabel signifikant waren. Alter, präoperative eGFR (errechnete glomeruläre Filtrationsrate), eGFR bei Entlassung und Operationsdauer wurden als lineare Variablen, das Vorliegen eines arteriellen Hypertonus oder jegliche postoperative Komplikation als dichotome Variable angewendet. Die Analysen erfolgten mit SPSS v25 (IBM, Armonk, USA), alle Tests waren zweiseitig, $p<0,05$ galt als signifikant.

\section{Ergebnisse}

\section{Gesamtergebnisse}

Die Nierenlebendspender waren meist Frauen im medianen Alter von 54 Jahren mit einem BMI von 25,3 kg/m² (• Tab. 1). Sie hatten keine relevanten Vorerkrankungen, $10 \%$ einen Nikotinabusus in der Vorgeschichte. In den meisten Fällen er- folgte die Nierenspende unter Ehepartnern, in $14(28 \%)$ weiteren Fällen von Eltern an Kinder und 11 (22\%) Fällen unter Geschwistern (s. • Abb. 1).

Die RDN dauerte im Median in $223,5 \mathrm{~min}$ bei einem Blutverlust von $50 \mathrm{ml}$ (• Tab. 2). Die warme Ischämiezeit betrug $2 \mathrm{~min}$, die KIZ $76 \mathrm{~min}$. Intraoperativ traten 3 Komplikationen auf, 1 (2\%) RDN wurde aufgrund massiver Adipositas und multiplen Trokardislokationen binnen der ersten 10 min nach offen konvertiert, die eigentliche $\mathrm{DN}$ erfolgte problemlos. In 2 (4\%) weiteren Fällen kam es zu Blutungen, einmal aufgrund einer Fehlfunktion eines Staplers, der schnitt, aber den Gefäßstumpf nicht verschloss. Diese und eine weitere Blutung aus einer Lumbalvene wurden robotisch beherrscht. Im postoperativen Verlauf trat eine Clavien-Dindo Grad 3a Komplikation auf, bei einem Ileus wurde eine Gastroskopie durchgeführt. Die mittlere Verweildauer betrug 5 Tage.

Die Entnahmeseite hatte keine Auswirkungen auf das operative Outcome, 
weder Operationszeit noch Komplikationsraten waren verschieden (s. ๑ Tab. 2). Lediglich die WIZ war bei den 10 (20\%) rechtsseitigen Nierenentnahmen kürzer ( 2 vs. $3 \mathrm{~min}, p<0,05$ ).

\section{Follow-up der Nierenfunktion}

Zum Zeitpunkt der Entlassung war die Nierenfunktion des Spenders signifikant schlechter (eGFR 88,5 vs. 56,$6 ; p<0,001$ ), danach blieb sie unverändert (• Abb. 2). Vor der Transplantation war kein $\mathrm{Pa}$ tient niereninsuffizient, nach RDN hatten fast die Hälfte ein CKD-Stadium $\geq 3 \mathrm{a}$ $(p<0,001, \bullet$ Tab. 3). Der Anteil niereninsuffizienter Patienten nahm in den Folgejahren tendenziell zu, wobei sich die Nierenfunktion nicht signifikant verschlechterte und erst bei 2 Patienten die RDN 10 Jahre zurückliegt.

In der multivariablen Regression war die präoperative Nierenfunktion sowohl für die postoperative Nierenfunktion (BWert $0,51, p<0,001$ ) und das Vorliegen einer Niereninsuffizienz prädiktiv (OR $0,9, p<0,01$, $\square$ Tab. 4). Ein höheres Spenderalter sowie das Vorliegen eines Hypertonus waren nur univariabel signifikant. Operative Parameter hatten keinen Einfluss auf die Nierenfunktion zum Zeitpunkt der Entlassung.

Bezüglich der einjährigen Nierenfunktion war nicht nur die präoperative Nierenfunktion (B-Wert 0,51, $p<0,01$ ), sondern auch das Lebensalter (B-Wert $-0,43, p<0,05)$ multivariabel signifikant (•Tab. 4). Das präoperative Vorliegen einer arteriellen Hypertonie sowie die postoperative Nierenfunktion waren nur univariabel prädiktiv. Für das Vorliegen einer Niereninsuffizienz $1 \mathrm{Jahr}$ nach Operation war ausschließlich die präoperative Nierenfunktion ein Prädiktor (OR 0,72, $p<0,05$ ).

\section{Diskussion}

In dieser Studie wurden retrospektiv die ersten 50 robotischen Donornephrektomien (RDN) eines urologischen Zentrums in Deutschland ausgewertet, wobei die erste RDN im Juni 2007 erfolgte. Das oberste Ziel einer DN ist die Sicherheit des Donors, da sich ein überdurchschnittlich Gesunder einem elek-

Urologe 2020 · 59:1512-1518 https://doi.org/10.1007/s00120-020-01302-w

(c) Der/die Autor(en) 2020

\section{P. Zeuschner $\cdot$ S. Siemer $\cdot$ M. Stöckle $\cdot$ M. Saar}

Die ersten 50 roboterassistierten Donornephrektomien. „Lessons learned"

\section{Zusammenfassung}

Hintergrund. Die minimal-invasive Donornephrektomie (DN) ist inzwischen operativer Standard, bezüglich der Rolle von roboterassistierten Verfahren gibt es bisher keinen Konsens.

Fragestellung. Die ersten 50 transperitonealen roboterassistierten Donornephrektomien (RDN) einer urologischen Universitätsklinik in Deutschland wurden retrospektiv ausgewertet.

Material und Methoden. Patientencharakteristika, intra- und postoperative Parameter wurden erfasst und die Nierenfunktion in einem 5-jährigen Follow-up ausgewertet. Signifikante Prädiktoren für die Nierenfunktion bei Entlassung und ein Jahr postoperativ wurden in einem multivariablen Regressionsmodell bestimmt.

Ergebnisse. Die RDN hat exzellente Ergebnisse mit niedriger Komplikationsrate, kurzer warmer (WIZ) und kalter Ischämiezeit (KIZ) sowie geringem Blutverlust und kurzer Patientenverweildauer. Die Seite der Nierenentnahme hat hierauf keine Auswirkungen. Nach RDN sind etwa 50\% der Spender formal niereninsuffizient, was aber zumeist ohne Relevanz ist, weil sich die Nierenfunktion der Spender im Follow-up nicht weiter verschlechtert. Die postoperative Nierenfunktion lässt sich bei der RDN mithilfe der präoperativen eGFR (errechnete glomeruläre Filtrationsrate) und dem Spenderalter sehr gut vorhersagen. Schlussfolgerungen. Die robotische DN stellt eine sehr gute Alternative zu anderen minimal-invasiven Operationsverfahren dar, die von Beginn an exzellente operative Ergebnisse ermöglicht.

Schlüsselwörter

Nierentransplantation - Nierenlebendspende . Minimal-invasive Chirurgie · Roboterassistiertes Operieren · Roboterassistierte Donornephrektomie

\section{The first 50 robot-assisted donor nephrectomies. Lessons learned}

\section{Abstract}

Background. Minimally invasive donor nephrectomy (DN) is considered the gold standard, but the role of robot-assisted surgery is still controversial.

Objectives. The first 50 robot-assisted DN (RDN) of a urologic transplant department in Germany were retrospectively analyzed. Materials and methods. Patient characteristics as well as intra- and postoperative surgical parameters were obtained. The kidney function of the donor was assessed within 5 years of follow-up. Predictors of postoperative kidney function at discharge and 1 year after RDN were estimated by multivariate regression analysis.

Results. RDN has an excellent surgical outcome with low complication rates, short warm (WIT) and cold ischemia time (CIT), little blood loss, and short patient stay. The side of donor nephrectomy does not affect surgical outcome. After RDN, $50 \%$ of donors suffer from mild to moderate renal insufficiency without further consequences, as their kidney function does not further decrease. Preoperative eGFR (estimated glomerular filtration rate) and donor age at surgery are the best predictors of postoperative kidney function after RDN.

Conclusions. Robot-assisted donor nephrectomy is an excellent alternative to other minimally invasive approaches rendering solid surgical results possible right from the start.

\section{Keywords}

Kidney transplantation - Living kidney donation - Minimally-invasive surgical procedures · Robot-assisted surgery $\cdot$ Robotassisted donor nephrectomy tiven Eingriff in altruistischer Intention unterzieht. Die Komplikationsrate der ersten 50 RDN war sehr gering mit 3 (6\%) intraoperativen Komplikationen inklusive einer Konversion nach offen wegen multipler Trokardislokationen bei Adi- positas. Die häufigsten Komplikationen bei RDN aber auch der LDN sind Blutungen [7]. Auch hier traten zwei Blutungen auf, eine aus der Lumbalvene und eine aus der A. renalis infolge eines defekten Staplersystems. Beide Blutungen konn- 


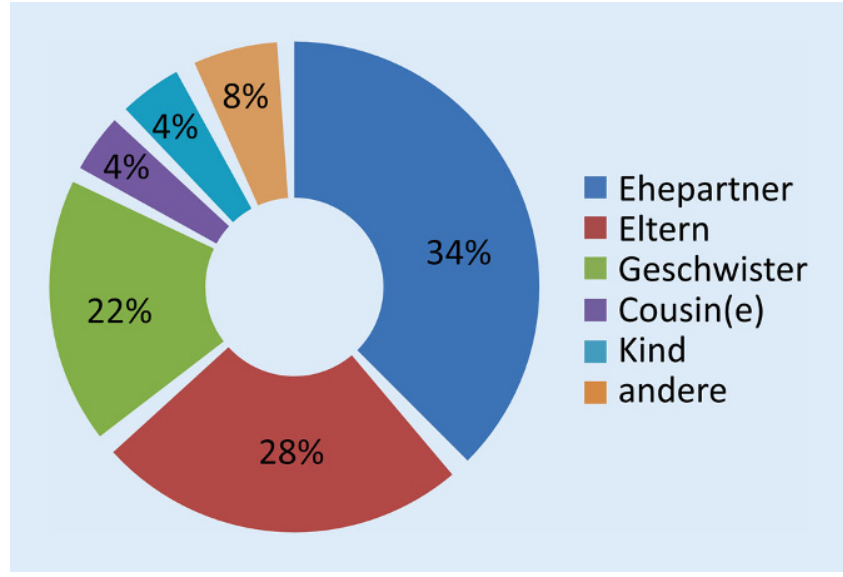

Abb. $1<$ Verwandtschaftsverhältnis des Spenders zum Empfänger ten robotisch kontrolliert werden und führten nicht zu Massenblutungen.

Die Versorgung des Gefäßstils bei der Donornephrektomie ist seit einer Warnung der FDA zum Einsatz von HemO-Lok-Clips bei der LDN im Jahr 2006 v. a. aus rechtlicher Sicht komplex geworden (• Abb. 3; [1]). Zuvor waren bei zwei Nierenspendern insuffizient schließende Clips abgerutscht, und die Patienten verbluteten [8]. Die FDA und auch die Deutsche Transplantationsgesellschaft warnten deshalb vor der Verwendung von Hem-O-Lok-Clips bei der LDN. Wir änderten unser Vorgehen und setzen fortan bei jeglicher roboterassistierten $\mathrm{Ne}$ phrektomie Staplersysteme ein, jedoch wie in anderen Kliniken mit erheblichen Problemen aufgrund vieler Fehlfunktionen, auch bei einer RDN [4]. Aus diesem Grund wurde sich dazu entschlossen, unter gewissen Vorsichtsmaßnahmen doch wieder Clips zu verwenden. Entscheidend ist wahrscheinlich, dass der Clip nicht direkt an der Aortenwand platziert wird, da ihn dort die kontinuierliche Gefäßpulsation zum Abrutschen bringt. Aus dem gleichen Grund sollte die Arterie nicht direkt am Clip, sondern mit einem Sicherheitsabstand von 2-3 mm abgetrennt werden. Dann ist das Risiko nicht größer als bei einer gewöhnlichen Nephrektomie, insbesondere rechtsseitig, wo regelhaft ein Stück weit entfernt von der Aortenwand abgesetzt wird. Als weitere Vorsichtsmaßnahme setzen wir zusätzlich einen zweiten Clip weiter peripher, eine Durchstechungsligatur dient als Abrutschsicherung. Auf diese Art und Weise haben wir bis heute keine negativen Erfahrungen bei der Verwendung von
Hem-O-Lok-Clips gemacht. Eine Metaanalyse konnte zudem keine Unterschiede zwischen Staplern und Clips hinsichtlich der Blutungskomplikations- oder Todesrate zeigen [19]. Alternativ kann auch auf Titanclips zurückgegriffen werden. Bei der Verwendung von Hem-O-LokClips sollten Nierenspender jedoch einer gesonderten Risikoaufklärung unterzogen werden, dies ist auch in anderen deutschen Transplantationszentren der Fall [6]. Ob eine gesonderte Risikoaufklärung in diesem Spannungsfeld auch zukünftig rechtlich haltbar ist, wird sich zeigen. Insofern sollte jedes Zentrum, das Donornephrektomien durchführt, für sich selbst entscheiden, welche Methode zur Gefäßkontrolle die Beste ist.

\section{Lesson \#1: Die Verwendung von Hem-O-Lok-Clips sollte nur mit Bedacht erfolgen}

Die Einführung minimal-invasiver Operationstechniken bei der $\mathrm{DN}$ führte weltweit zunächst zu weiteren Problemen. Metaanalysen zeigten, dass bei der LDN rechtsseitige Nieren aufgrund ihrer komplexeren Gefäßanatomie bei der Lebendspende signifikant häufiger eine delayed graft function (DGF), höhere Graft-Thromboserate sowie insgesamt ein schlechteres Graftüberleben als linke Nieren hatten $[15,18]$. Diese Unterschiede waren gering und in spezialisierten Zentren (fast) nicht messbar, weswegen die EAU Guidelines bei der LDN heute von einer gleichwertigen Sicherheit im Vergleich zur ODN ausgehen [5]. Das Outcome der ersten $50 \mathrm{RDN}$ zeigt im Seitenvergleich keine signifikanten Un- terschiede (•Tab. 2), lediglich die WIZ war bei den linksseitigen Nierenentnahmen geringfügig länger (3 vs. $2 \mathrm{~min}$, $p<0,05)$. Dies ist mit einem Lernkurveneffekt zu erklären, denn rechtsseitige Nieren wurden erst bei steigender Erfahrung mit der RDN durchgeführt. Dass sich die restlichen Parameter nicht unterscheiden, ist am ehesten darauf zurückzuführen, dass sich die Vorzüge des robotischen Operierens im Vergleich zur Laparoskopie besonders in komplexen Situationen zeigt: bestimmte Manöver verlangen dem Operateur beim Laparoskopieren deutlich mehr Geschick ab als am Roboter - unsere Blutungskomplikationen mussten so nicht konvertiert werden. Die Studienlage ist diesbezüglich nicht eindeutig, einige Arbeiten zeigen Unterschiede zwischen rechtsund linksseitiger RDN, andere nicht [6, 26]. Aus rein operativer Sicht spielt die Organseite für die RDN unserer Erfahrung nach eine untergeordnete Rolle, vielmehr sollten Nierenfunktion oder anatomische Besonderheiten für die Seitenwahl maßgeblich sein. Der Operateur sollte jedoch im Zweifelsfall die operative Technik den Gegebenheiten anpassen und mehrere beherrschen, von ODN über LDN oder auch die RDN.

\section{Lesson \#2: Für die RDN ist die Organseite unerheblich}

Die mediane Operationszeit von 223,5 min ist im Vergleich zu anderen Arbeiten etwa 30-60 min länger [28]. Mehrere Gruppen beschreiben bei der RDN eine Lernkurve, da die Operationszeit mit steigender Erfahrung abnimmt [13]. Ganz unabhängig davon sind die Operationszeiten bei Horgan et al. [13] schon bei den ersten Fällen kürzer (201 $\pm 42 \mathrm{~min})$, denn nicht mangelnde Erfahrung, sondern die Abläufe im Universitätsklinikum Homburg führen zu einer längeren Operationszeit. Um eine kurze KIZ zu ermöglichen, werden Spender und Empfänger simultan in zwei Sälen von zwei Teams operiert. Die Niere wird erst dann abgesetzt, wenn das Transplantationsteam sicher ist, dass die Transplantation wie geplant möglich ist. Dies kann dazu führen, dass ein Team auf das andere warten muss, was die Operationszeit verlängert, aber die KIZ 

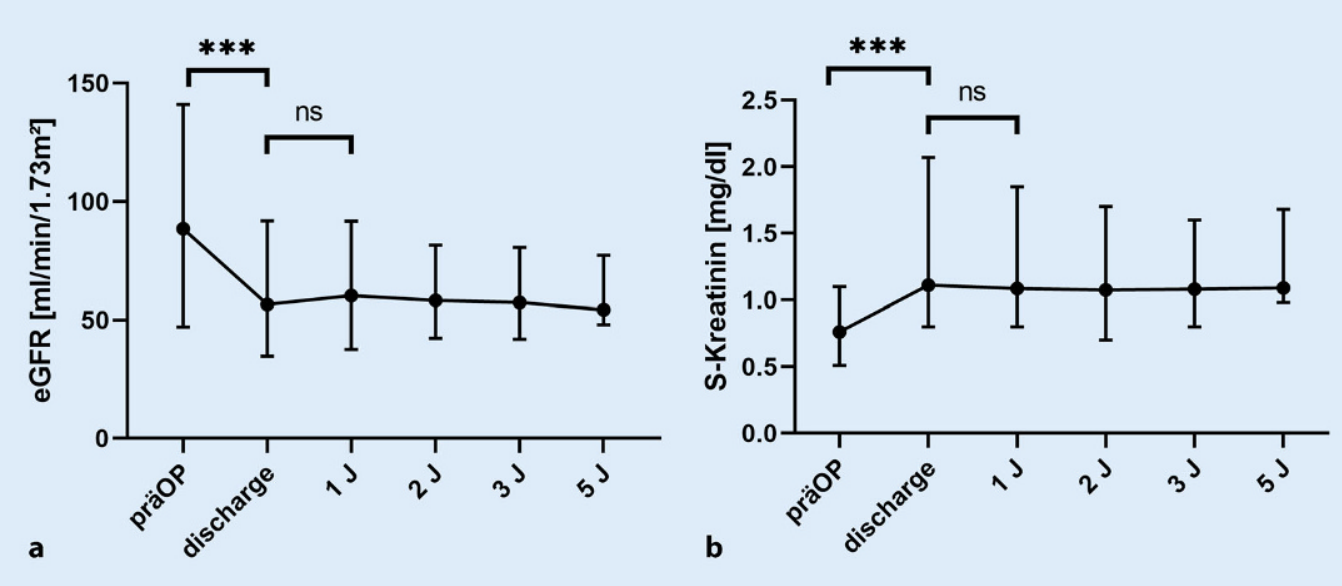

Abb. $2 \varangle$ Follow-up der Nierenfunktion: a eGFR (errechnete glomeruläre Filtrationsrate), b S-Kreatinin
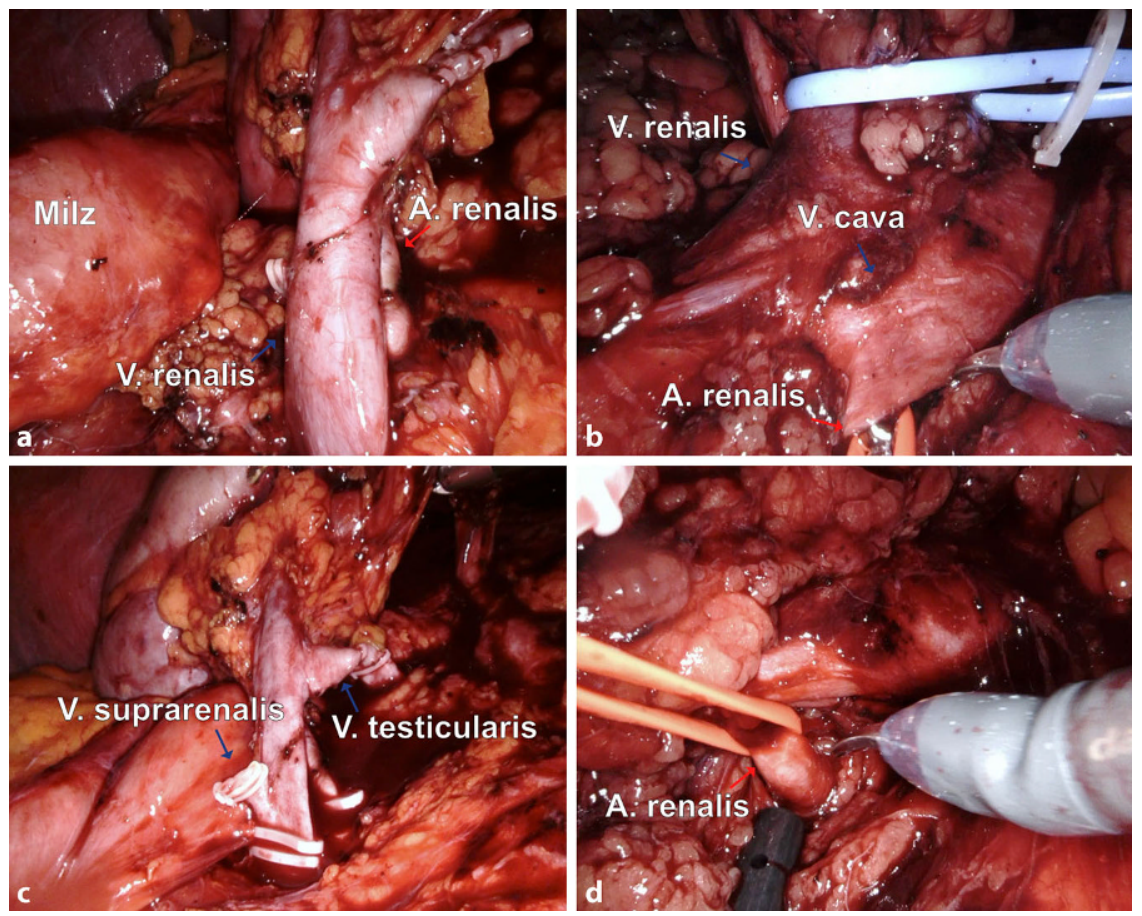

Abb. $3 \Delta$ Die Versorgung einer rechtsseitigen Niere $(\mathbf{b}, \mathbf{d})$ ist im Vergleich zur linken Niere $(\mathbf{a}, \mathbf{c})$ aufgrund der Gefäßanatomie anspruchsvoller. Die A. renalis dextra wurde hier interaortocaval angeschlungen (d)

signifikant verkürzt. Die französischen Kollegen aus Nancy erreichen nach 150 RDN eine mediane Operationszeit von $176 \pm 23 \mathrm{~min}$, aber nehmen eine fast dreimal so lange KIZ von $206 \pm 71 \mathrm{~min}$ in Kauf, weil sie die Patienten nacheinander operieren [17]. Auch wenn eine längere KIZ kein signifikanter Prädiktor für eine postoperativ schlechtere Nierenfunktion ist, zeigt sich tendenziell ein schlechteres Outcome, wenn die KIZ $2 \mathrm{~h}$ übersteigt $[9,22]$. Während der „Wartezeit“ auf das Absetzen der Niere wird das Pneumoperitoneum bei uns vollständig abgelassen, damit sich die Spenderniere erholen kann. In verschiedenen Arbeiten konnte gezeigt werden, dass das Pneumoperitoneum negative Auswirkungen auf die Mikrozirkulation hat und daher die Niere(n) schädigen kann [23, 27]. Möglicherweise genau infolge dieser „Erholungspause“ setzt in den meisten Fällen bereits intraoperativ die Urinproduktion bei der Transplantation ein, die seit 2016 auch robotisch durchgeführt wird [30]. Um den Empfängern optimale Voraussetzungen für eine bestmögliche Nierenfunktion $\mathrm{zu}$ bieten, werden wir zukünftig an dieser Vorgehensweise festhalten.

\section{Lesson \#3: Sorgfalt geht vor}

Häufig wird auch gegen die RDN angeführt, die WIZ sei im Vergleich zur LDN länger, sie beträgt hier $3 \mathrm{~min}$ ( $\bullet$ Tab. 2; [3]). Dies ist damit zu erklären, dass bei der RDN der Operationsroboter vom Situs abgedockt werden muss, beim laparoskopischen Vorgehen nicht. Wir verwenden schon seit Längerem einen GelPort $^{\circledR}$-Trokar, dessen Kappe problemlos und schnell entfernt werden kann. Ganz unabhängig davon ist es sehr unwahrscheinlich, dass kleine Unterschiede von nur wenigen Minuten WIZ überhaupt Auswirkungen auf die postoperative Nierenfunktion haben. In der Nierentumorchirurgie konnte gezeigt werden, dass eine WIZ von unter 20 min nur eine geringe Auswirkung auf das Organ hat [10]. Auch für die Nierentransplantation wurde belegt, dass erst eine WIZ $>45$ min die Organfunktion bei Nierenlebendspenden signifikant verschlechtert, darunter konnten keine negativen Auswirkungen festgestellt werden [11]. Insofern ist eine WIZ von 2-3 min ein exzellentes operatives Ergebnis.

\section{Lesson \#4: Die WIZ ist auch bei RDN kurz}

Die postoperative Nierenfunktion des Spenders ist im Vergleich zur präoperativen Situation schlechter (• Abb. 2). Im Follow-up nahm die Nierenfunktion 
Tab. 4 Signifikante Einflussfaktoren auf die Nierenfunktion zum Zeitpunkt der Entlassung und im einjährigen Follow-up (nur multivariabel)

\begin{tabular}{|c|c|c|c|c|}
\hline & \multicolumn{2}{|l|}{ eGFR Entlassung } & \multicolumn{2}{|c|}{ Niereninsuffizienz Entlassung } \\
\hline & B-Wert (95\%-KI) & $p$-Wert & OR $(95 \%-K I)$ & $p$-Wert \\
\hline Alter & - & n.s. & - & - \\
\hline Hypertonus & - & n.s. & - & n.s. $(0,07)$ \\
\hline eGFR präoperativ & $0,51(0,31 ; 0,71)$ & $<0,001$ & $0,9(0,86 ; 0,97)$ & $<0,01$ \\
\hline Operationsdauer & - & - & - & - \\
\hline \multirow[t]{3}{*}{ Komplikationen } & - & - & - & - \\
\hline & \multicolumn{2}{|l|}{ eGFR $1 \mathrm{~J}$} & \multicolumn{2}{|c|}{ Niereninsuffizienz $1 \mathrm{~J}$} \\
\hline & B-Wert (95\% Kl) & $p$-Wert & OR $(95 \% \mathrm{KI})$ & $p$-Wert \\
\hline Alter & $-0,43(-0,7 ;-0,16)$ & $<0,05$ & & n.s. \\
\hline Hypertonus & - & n.s. & - & - \\
\hline eGFR präoperativ & $0,51(0,21 ; 0,8)$ & $<0,01$ & $0,72(0,53 ; 0,98)$ & $<0,05$ \\
\hline eGFR Entlassung & - & n.s. & - & n.s. \\
\hline Operationsdauer & - & - & - & - \\
\hline Komplikationen & - & - & - & - \\
\hline
\end{tabular}

nicht weiter $a b$, aber verbesserte sich auch nicht mehr. Damit sind fast $50 \%$ der Nierenspender nach RDN formell niereninsuffizient. Auch wenn ehemals Arbeiten titelten, die Nierenfunktion von Nierenlebendspendern sei im Vergleich zur Allgemeinbevölkerung langfristig gleich, so trifft dies in der Realität nicht zu [14]. Das Risiko für terminale Niereninsuffizienz ist in gematchten Kollektiven erhöht, weil Nierenspender gesünder sind als die Normalbevölkerung - trotzdem ist das Risiko immer noch als gering einzuschätzen [21]. Im eigenen Kollektiv ist bisher kein einziger Patient niereninsuffizient geworden, die Nierenfunktion der Spender hat sich seit der RDN während des Follow-ups auch nicht verschlechtert (• Abb. 2).

In einer Regressionsanalyse sind Patientenalter, arterielle Hypertonie und präoperative Nierenfunktion gute Prädiktoren für die Nierenfunktion bei Entlassung und im einjährigen Follow-up nach RDN (• Tab. 4). In der multivariablen Regression war jedoch nur noch die präoperative Nierenfunktion signifikant, bei der einjährigen eGFR auch das Alter (BWert $-0,43, p<0,05)$. In diesem Zusammenhang entwickelten Benoit et al. [2] 2017 ein Vorhersagemodell für die postoperative Nierenfunktion ein Jahr nach LDN [16]. Dieses Modell lässt sich sehr gut auf die RDN übertragen, weil bei Benoit et al. [2] ebenso nur präopera- tive eGFR und das Donoralter Prädiktoren für die einjährige eGFR sind. Erstaunlicherweise unterscheiden sich die Koeffizienten nur gering: bei der RDN errechnet sich die eGFR ein Jahr postoperativ mit $32,29+(0,56 \times$ präoperative eGFR) - (0,44× Alter), bei der LDN nach Benoit et al. mit 31,17+ (0,52 $\times$ präoperative eGFR) - (0,31 $\times$ Alter $)$. Da operative Parameter auch bei der RDN keinen Einfluss auf die postoperative Nierenfunktion haben, ist das Operationsverfahren unwichtig.

\section{Lesson \#5: Präoperative Nierenfunktion und Donoralter zählen}

Diese Arbeit ist nicht frei von Limitationen. Es handelt sich zwar um die größte monozentrische Kohorte einer urologischen deutschen Transplantationsklinik, jedoch spiegelt sie nur eine Perspektive wider, zumal am Universitätsklinikum Homburg fast keine laparoskopischen Eingriffe durchgeführt werden. Es handelt sich somit um Ergebnisse eines großen robotischen Zentrums, die nicht zwangsläufig auf jede Klinik übertragbar sein müssen.

\section{Fazit für die Praxis}

- Die robotische Donornephrektomie (RDN) hat sich mit weltweit mehr
Infobox Mehr Informationen zum Thema

DSO-Jahresbericht: https://tinyurl.com/ y73h8bc6

- DDVZ: https://tinyurl.com/yde4hjp4

- AK NTx: https://www.nierentransplantation.com/

als 1000 Eingriffen als operatives Standardverfahren in spezialisierten Zentren etabliert.

- Die ersten 50 RDN an einem deutschen urologischen Zentrum haben exzellente Ergebnisse mit kurzer WIZ (warme Ischämiezeit) und KIZ (kalte Ischämiezeit).

- Die RDN stellt wie die laparoskopische DN (LDN) besondere Anforderungen hinsichtlich der Versorgung der Nierengefäße.

- Die rechtsseitige RDN hat im Gegensatz zu LDN-Serien, die anfänglich rechts schlechtere Ergebnisse als links zeigten, von Beginn an ein gleichwertiges operatives Outcome.

- Selbst wenn die RDN technisch womöglich einfacher als die LDN ist, steht sie vor denselben komplexen organisatorischen Anforderungen im Spannungsfeld medizinscher, ethischer aber auch ökonomischer Faktoren.

- Ein hoher Anteil an Nierenspendern ist nach RDN formell niereninsuffizient, wobei präoperative Nierenfunktion und Lebensalter hierfür maßgeblich sind - dies hat in der Praxis zumeist keine große Relevanz.

\section{Korrespondenzadresse

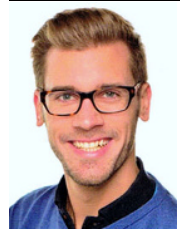 \\ Dr. med. Philip Zeuschner \\ Klinik für Urologie und Kinderurologie, Universitätsklinikum des \\ Saarlandes und Medizinische Fakultät der Universität des Saarlandes Kirrberger Straße 100, 66421 Homburg/Saar, Deutschland philip.zeuschner@uks.eu}

Funding. Open Access funding provided by Projekt DEAL. 


\section{Einhaltung ethischer Richtlinien}

Interessenkonflikt. P. Zeuschner, M. Stöckle und $M$. Saar geben an, dass kein Interessenkonflikt besteht. S. Siemer: Procter für Intuitve Surgical Systems, Vortragshonorare für BMS, Pfizer.

Alle beschriebenen Untersuchungen am Menschen oder an menschlichem Gewebe wurden mit Zustimmung der zuständigen Ethikkommission, im Einklang mit nationalem Recht sowie gemäß der Deklaration von Helsinkivon 1975 (in der aktuellen, überarbeiteten Fassung) durchgeführt. Von allen beteiligten Patienten liegt eine Einverständniserklärung vor. Es liegt ein Ethikvotum (Nr. 106/18) der Ethik-Kommission der Ärztekammer des Saarlandes vor.

Open Access. Dieser Artikel wird unter der Creative Commons Namensnennung 4.0 International Lizenz veröffentlicht, welche die Nutzung, Vervielfältigung, Bearbeitung, Verbreitung und Wiedergabe in jeglichem Medium und Format erlaubt, sofern Sie den/die ursprünglichen Autor(en) und die Quelle ordnungsgemäß nennen, einen Link zur Creative Commons Lizenz beifügen und angeben, ob Änderungen vorgenommen wurden.

Die in diesem Artikel enthaltenen Bilder und sonstiges Drittmaterial unterliegen ebenfalls der genannten Creative Commons Lizenz, sofern sich aus der Abbildungslegende nichts anderes ergibt. Sofern das betreffende Material nicht unter der genannten Creative Commons Lizenz steht und die betreffende Handlung nicht nach gesetzlichen Vorschriften erlaubt ist, ist für die oben aufgeführten Weiterverwendungen des Materials die Einwilligung des jeweiligen Rechteinhabers einzuholen.

Weitere Details zur Lizenz entnehmen Sie bitte der Lizenzinformation aufhttp://creativecommons.org/ licenses/by/4.0/deed.de.

\section{Literatur}

1. Administration USFD (2006) Class 2 device recall Weck Hemolok Endo 5 automatic clip applier with medium polymer ligating clips. https://www. accessdata.fda.gov/scripts/cdrh/cfdocs/cfres/res. cfm?id=45877. Zugegriffen: 29.05 .2020

2. Benoit T, Game X, Roumiguie M et al (2017) Predictive model of 1-year postoperative renal function after living donor nephrectomy. Int Urol Nephrol 49:793-801

3. Bhattu AS, Ganpule A, Sabnis RB et al (2015) Robot-assisted laparoscopic donor nephrectomy vs standard laparoscopic donor nephrectomy: a prospective randomized comparative study. JEndourol 29:1334-1340

4. Breda A, Veale J, Liao J et al (2007) Complications of laparoscopic living donor nephrectomy and their management: the UCLA experience. Urology 69:49-52

5. Breda KB, Figueiredo A, Lledó García E, Olsburgh J, Regele H, Boissier R, Fraser Taylor C, Hevia V, Rodríguez Faba O, Zakri RH (2020) EAU guidelines on renal transplantation

6. Brunotte M, Rademacher S, Weber J et al (2020) Robotic assisted nephrectomy for living kidney donation (RANLD) with use of multiple locking clips or ligatures for renal vascular closure. Ann TransI Med 8:305
7. Friedersdorff F, Kothmann L, Manus P et al (2016) Long-term donor outcomes after pure laparoscopic versus open living donor nephrectomy: focus on pregnancy rates, hypertension and quality of life. Urol Int 97:450-456

8. Friedman AL, Peters TG, Ratner LE (2012) Regulatory failure contributing to deaths of live kidney donors. Am JTransplant 12:829-834

9. Gill J, Rose C, Joffres Y et al (2017) Cold ischemia time up to 16 hours has little impact on living donor kidney transplant outcomes in the era of kidney paired donation. Kidney Int 92:490-496

10. Ginzburg S, Uzzo R, Walton J et al (2015) Residual parenchymal volume, not warm Ischemia time, predicts ultimate renal functional outcomes in patients undergoing partial nephrectomy. Urology 86:300-305

11. Hellegering J, Visser J, Kloke HJ et al (2012) Deleterious influence of prolonged warm ischemia in living donor kidney transplantation. Transplant Proc 44:1222-1226

12. Horgan S, Vanuno D, Sileri Petal (2002) Robotic-assisted laparoscopic donor nephrectomy for kidney transplantation. Transplantation 73:1474-1479

13. Horgan S, GalvaniC, Gorodner MVetal (2007) Effect of robotic assistance on the "learning curve" for laparoscopic hand-assisted donor nephrectomy. Surg Endosc 21:1512-1517

14. Ibrahim HN, Foley R, Tan L et al (2009) Long-term consequences of kidney donation. N Engl J Med 360:459-469

15. Khalil A, Mujtaba MA, Taber TE et al (2016) Trends and outcomes in right vs. left living donor nephrectomy: an analysis of the OPTN/UNOS database of donor and recipient outcomes-should we be doing more right-sided nephrectomies? Clin Transplant 30:145-153

16. Kulik U, Gwiasda J, Oldhafer F et al (2017) External validation of a proposed prognostic model for the prediction of 1-year postoperative eGFR after living donor nephrectomy. Int Urol Nephrol 49:1937-1940

17. Leblanc $L$, Lagrange $F$, Ladriere $M$ et al (2019) Robotic-assisted laparoscopic living donor nephrectomy: study in donors and recipients from 155 cases. Prog Urol 29:596-602

18. Liu N, Wazir R, Wang J et al (2014) Maximizing the donor pool: left versus right laparoscopic live donor nephrectomy - systematic review and meta-analysis. Int Urol Nephrol 46:1511-1519

19. Liu Y, Huang Z, Chen Y et al (2018) Staplers or clips?: a systematic review and meta-analysis of vessel controlling devices for renal pedicle ligation in laparoscopic live donor nephrectomy. Medicine 97:e13116

20. Luke PP, Aquil S, Alharbi B et al (2018) First Canadian experience with robotic laparoendoscopic single-site vs. standard laparoscopic living-donor nephrectomy: a prospective comparative study. Can Urol Assoc J 12:E440-E446

21. Muzaale AD, Massie AB, Wang MC et al (2014) Risk of end-stage renal disease following live kidney donation. JAMA 311:579-586

22. Nath J, Hodson J, Canbilen SW et al (2016) Effect of cold ischaemia time on outcome after living donor renal transplantation. Br JSurg 103:1230-1236

23. Ozdemir-Van Brunschot DM, Van Laarhoven KC, Scheffer GJ et al (2016) What is the evidence for the use of low-pressure pneumoperitoneum? A systematic review. Surg Endosc 30:2049-2065

24. Pietrabissa A, Abelli M, Spinillo A et al (2010) Robotic-assisted laparoscopic donor nephrectomy with transvaginal extraction of the kidney. Am J Transplant 10:2708-2711
25. Ratner LE, Ciseck LJ, Moore RG, Cigarroa FG, Kaufman HS, Kavoussi LR (1995) Laparoscopic live donor nephrectomy. Transplantation 60(9):1047-1049

26. Renoult E, Hubert J, Ladriere M et al (2006) Robot-assisted laparoscopic and open live-donor nephrectomy: a comparison of donor morbidity and early renal allograft outcomes. Nephrol Dial Transplant 21:472-477

27. Sodha S, Nazarian S, Adshead JM et al (2015) Effect of pneumoperitoneum on renal function and physiology in patients undergoing robotic renal surgery. Curr Urol 9:1-4

28. Wang H, Chen R, Li T et al (2019) Robot-assisted laparoscopic vs laparoscopic donor nephrectomy in renal transplantation: a meta-analysis. Clin Transplant 33:e13451

29. Wilson CH, Sanni A, Rix DA et al (2011) Laparoscopic versus open nephrectomy for live kidney donors. Cochrane Database Syst Rev. https://doi.org/10. 1002/14651858.CD006124.pub2

30. Zeuschner P, Siemer S, Stockle M (2020) Robotassisted kidney transplantation. Urologe A 59:3-9 